\title{
Encapsulation Methods for Solid Radionuclide Production Targets at a Medium-Energy Cyclotron Facility
}

\author{
Gideon Steyn $^{1, \mathrm{a}), \mathrm{b})}$, Christiaan Vermeulen ${ }^{2, \mathrm{c})}$ and Eugene Isaacs ${ }^{1, \mathrm{~d})}$ \\ ${ }^{1}$ iThemba LABS, P.O. Box 722, Somerset West, 7129, South Africa \\ ${ }^{2}$ Paul Scherrer Institute, Villigen-PSI, Switzerland \\ ${ }^{a}$ Corresponding author: deon@tlabs.ac.za \\ ${ }^{b)}$ URL: http://www.tlabs.ac.za \\ c) etienne.vermeulen@psi.ch \\ d)eisaacs@tlabs.ac.za
}

\begin{abstract}
The techniques employed at iThemba LABS for the encapsulation of solid radionuclide production targets, based on cold indentation welding, electron beam welding and laser welding, are described. Some aspects of the target holders and cooling requirements to bombard targets in a tandem configuration with a $66 \mathrm{MeV}$ proton beam, with intensities up to nominally $250 \mathrm{~A}$, are also briefly discussed. These techniques are inter alia suitable for a production regimen compatible with the new generation of commercial, high-intensity $70 \mathrm{MeV}$ cyclotrons.
\end{abstract}

\section{INTRODUCTION}

A significant percentage of solid targets for the production of radionuclides with an accelerator can be broadly classified into two categories. The first type employs a water-cooled backing material (or substrate) onto which the target material has been deposited. The target surface is usually slanted with respect to the beam axis in order to reduce the beam power density, thereby facilitating the bombardment with a beam of higher intensity. As an example, Arzumanov et al. [1] describe internal cyclotron targets consisting of copper substrates onto which various metallic target materials have been deposited by means of electrodeposition. The beam incidence angle in that case is $18^{\circ}$. This concept can also be adopted for external targetry. A recent study by Gelbart and Johnson [2], for example, describes an external target station that receives the beam at an incidence angle of $7^{\circ}$ onto targets designed to withstand beam powers of up to $50 \mathrm{~kW}$.

The second type of solid target typically has a circular disc or flat cylindrical shape, with the beam normal to the axial surface and usually swept (or wobbled) across the target surface to reduce the beam power density. These targets are completely surrounded by fast-flowing cooling water during bombardment. Target materials that need protection from the cooling water are encapsulated (or clad) with a metal that has a relatively high melting point. Various such combinations have been described in the literature. While the cold target materials are normally in a solid state, they are likely to become partially, or even completely, molten under bombardment conditions. The target capsule serves as a barrier to the cooling water as well as to contain the target material and to maintain its shape, should melting occur. Such targets are extensively utilized at e.g. the $100 \mathrm{MeV}$ Isotope Production Facility (IPF) at Los Alamos National Laboratory for the large-scale production of radionuclides [3, 4]. This approach is also followed at iThemba LABS, South Africa, for the routine production of radionuclides with a $66 \mathrm{MeV}$ proton beam [5].

The main aim of this paper is to discuss the various methods of target encapsulation employed at iThemba LABS, based on cold indentation welding, electron beam (EB) welding and laser welding. A brief discussion on target holders and tandem targetry is also presented. 


\section{ENCAPSULATION USING COLD INDENTATION WELDING}

Cold indentation welding is a particular form of cold pressure welding, where no external heating is applied and no liquid or molten phase is present at the weld joint. In order to obtain bonding, joint plastic deformation of the two metal parts at the weld joint is required [6-8]. For this purpose, a hydraulic press is used. The two parts of a capsule are bonded together inside a punch and die set to which a uniaxial pressure is applied. An indentation is formed along the flanges of the two metal components, resulting in a surface expansion of the bonding surface. Bonding is not obtained until a certain critical surface expansion has been reached, depending on the type of metal used and the preparation of the contact surfaces prior to the application of pressure.

A 300 ton hydraulic press (see Fig. 1) was designed and constructed at iThemba LABS, providing the capability to make solid target discs by powder compaction as well as encapsulating them afterwards, if required. The punch and die sets required for these actions have also been manufactured in-house [9].

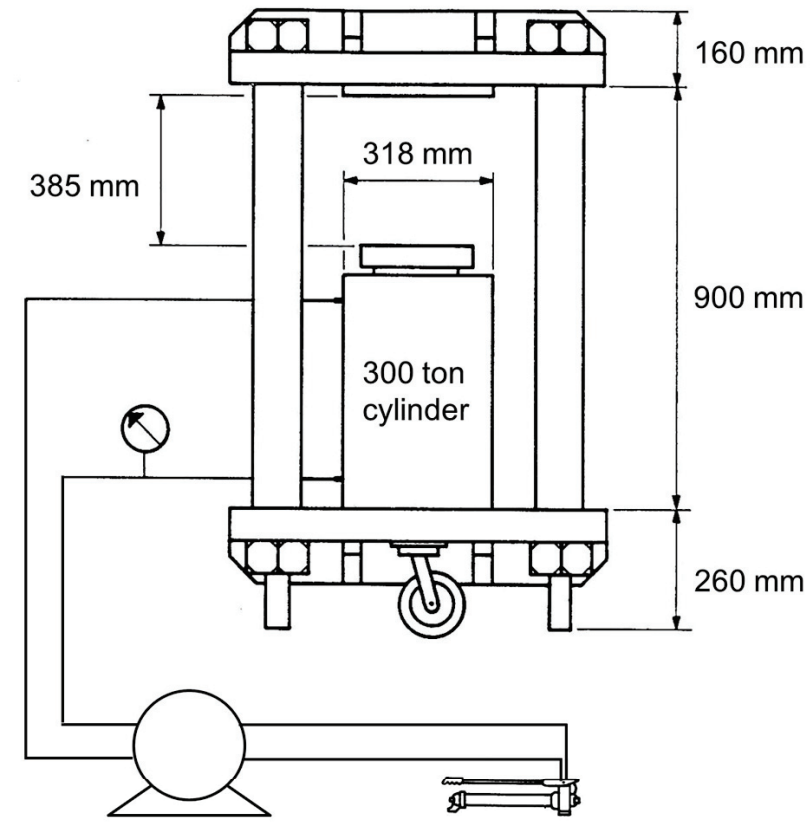

Hydraulic power pack

Hydraulic hand pump

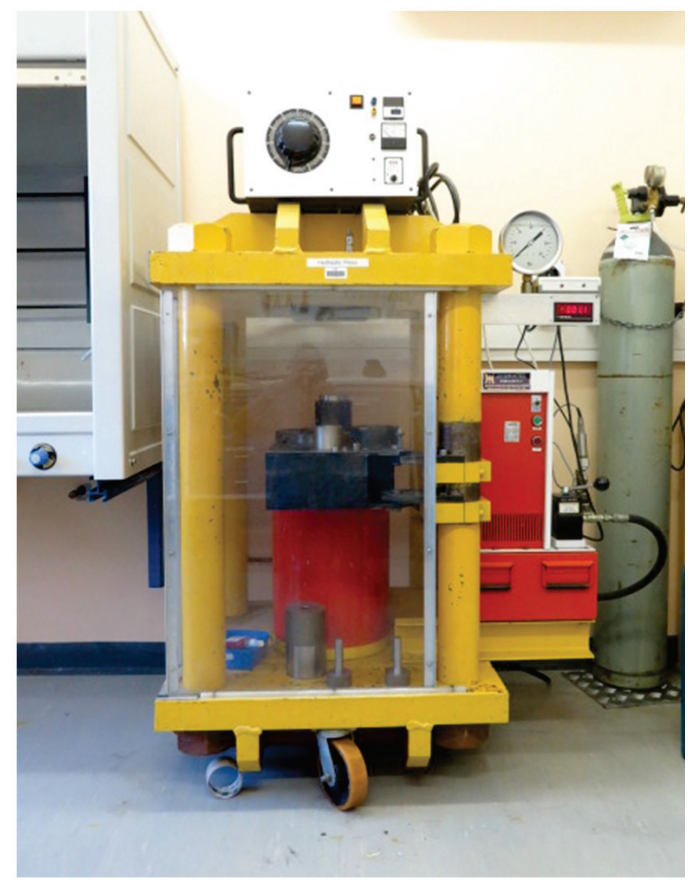

FIGURE 1. Schematic diagram and photograph of the 300 ton hydraulic press facility.

Figure 2 (a) shows a diagram of one of the punch and die sets used for cold indentation welding. The pieces of the set are both made of a Böhler steel, K107, hardened to 65 Rockwell C. Also shown in Fig. 2 is a diagram of a typical weld seam as well as photographs of some completed targets. Such targets have been produced at iThemba LABS for nearly three decades. Aluminium is mostly used as the capsule material but the method has also been demonstrated to work equally well with copper capsules. The punch and die set is normally connected to a fore-vacuum pump in order to remove the air from the capsule prior to the welding. Many different target materials have been sealed in Al capsules over the years, some for routine production purposes and others for experimental purposes. It is mainly used to protect compressed non-metals (such as compacted salt targets and/or other hygroscopic materials) from the cooling water. Some metals and metalloids proved to behave badly in direct contact with the cooling water, e.g. Mn and Ge. These types of target materials are therefore also encapsulated. The method is very versatile and is still extensively utilized in our laboratory. It was found that harder grades of Al that responds well to heat treatment, such as alloy grades 6061 or 6082 , give the best results.

Two vitally important requirements for this welding method to succeed are that the aluminium should be annealed and that the flange surfaces be thoroughly cleaned from oxides immediately prior to welding [10]. The capsules are typically annealed at $450^{\circ} \mathrm{C}$ for 30 minutes and allowed to cool in air to room temperature. This treatment effectively 
(a)
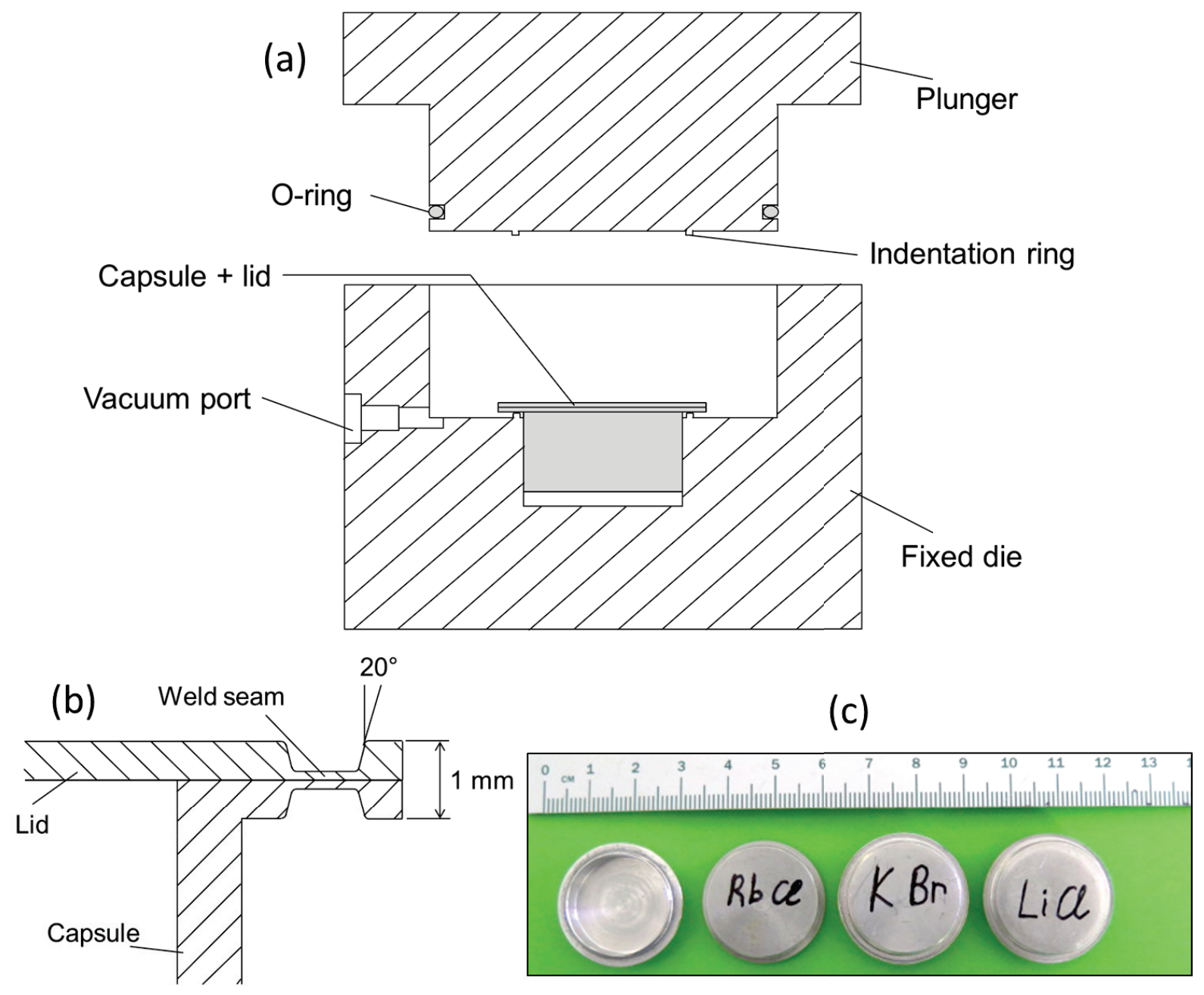

FIGURE 2. (a) Schematic diagram of a typical punch (or plunger) and die set for encapsulating targets in aluminium by means of the cold indentation welding technique. (b) Close-up view of a weld seam. (c) The two parts of an $\mathrm{Al}$ capsule for a $\mathrm{RbCl}$ target before welding as well as sealed $\mathrm{KBr}$ and $\mathrm{LiCl}$ targets, respectively.

relieves the residual stresses induced by the machining. The surface oxide is removed by wire brushing. A $0.5 \mathrm{~mm}$ strand rotary stainless steel wire brush is used for this purpose. The time between oxide removal and loading of the target into the punch and die set for welding is typically less than 1 minute.

Two parameters that are often quoted in conjunction with cold pressure welding applications described in the literature are the reduction, $R$, and the corresponding surface expansion, $X$. In the case of indentations on two joined flanges, $R$ will be the ratio of the total indented depth and the original total flange thickness. Several studies reported the bond shear strength as either a function of the reduction or the surface expansion. In key studies by Zhang and Bay [11, 12] on Al-Al bonding, it was shown that welding typically starts at a nominal reduction of $R=0.4$ but maximum bond shear strength is obtained closer to $R=0.8$. Experiences at iThemba LABS are very much in agreement with the results of Zhang and Bay. Consequently, the punch and die sets have been designed for a reduction of $R=0.8$. As shown in Fig. 2 (b), with a total flange thickness of $1 \mathrm{~mm}$, the thickness of the weld seam is only $0.2 \mathrm{~mm}$. This may at first appear to seriously weaken the welded joint; nevertheless, these welds are surprisingly strong. Burst tests

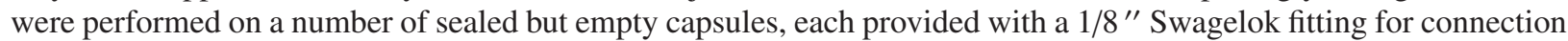
to the regulator of a nitrogen gas cylinder. The gas pressure was slowly increased until rupture occurred. The weakest region was indeed found to be along the weld seam but the rupture pressures were found to be quite high, between 17 and 23 bar. These results were collected on capsules made for targets with a diameter of $20 \mathrm{~mm}$. Helium leak tests 
were also carried out on 15 samples. All were found to be leak tight. It was also found that the speed of compression (e.g. by slowly pressing down the plunger or performing a rapid, near impact-like compression) makes no noticeable difference to the weld joint or integrity of the target capsule.

Scanning electron micrographs (see e.g. Ref. [7]) have revealed the mechanism of weld formation. At a critical point in the evolution of the indentation (plastic deformation of the metal and surface expansion) cracks appear in the two surface layers at the contact region. As further pressure is applied, extrusion of virgin metal through these cracks is initiated. Finally, significant contact of extruded virgin metal through the original surface layers leads to bonding and weld formation.

Most of the targets encapsulated by means of the cold indentation welding technique are for the production of radionuclides for medical use, but not all. One notable exception was the first successful experiment ever to measure the cluster radioactive decay of ${ }^{223} \mathrm{Ac}$ by ${ }^{14} \mathrm{C}$ and ${ }^{15} \mathrm{~N}$ emission $[13,14]$. The relevant reaction is ${ }^{232} \mathrm{Th}(\mathrm{p}, 6 \mathrm{n}){ }^{227} \mathrm{~Pa} \rightarrow{ }^{223} \mathrm{Ac}+\alpha$. By some luck, enough Th target material could be found to produce ten solid metallic target discs, each $4.7 \mathrm{~g} / \mathrm{cm}^{2}$ thick with a nominal mass of $8 \mathrm{~g}$. These target discs were encapsulated in $\mathrm{Al}$ (see Fig. 3). Several targets were used to develop a rapid radiochemical method to quantitatively separate the produced Pa from the Th matrix. Targets were typically bombarded with a $66 \mathrm{MeV}$ proton beam of $80 \mu \mathrm{A}$ for 2 hours. Dry sources were prepared from the separated $\mathrm{Pa} ;{ }^{227} \mathrm{~Pa}\left(T_{1 / 2}=38.3 \mathrm{~min}\right)$ feeds ${ }^{223} \mathrm{Ac}\left(T_{1 / 2}=2.1 \mathrm{~min}\right)$ by means of $\alpha$ decay. Solidstate nuclear track detectors (SSNTD) exposed to the emissions from these sources were analysed for ${ }^{14} \mathrm{C}$ and ${ }^{15} \mathrm{~N}$ tracks. Several hundred ${ }^{14} \mathrm{C}$ events were detected, providing a branching ratio consistent with the ${ }^{223} \mathrm{Ac} \rightarrow{ }^{14} \mathrm{C}+{ }^{209} \mathrm{Bi}$ decay channel being dominated by a favoured ground state to ground state transition. No ${ }^{15} \mathrm{~N}$ events were found, thus only an upper limit for the branching ratio of the ${ }^{223} \mathrm{Ac} \rightarrow{ }^{15} \mathrm{~N}+{ }^{208} \mathrm{~Pb}$ decay channel could be determined. Nevertheless, this result was found to be consistent with theoretical predictions for an unfavoured transition. Thus, in this case, the infrastructure and methods typical for a radionuclide production facility also proved useful for an interesting nuclear physics experiment.

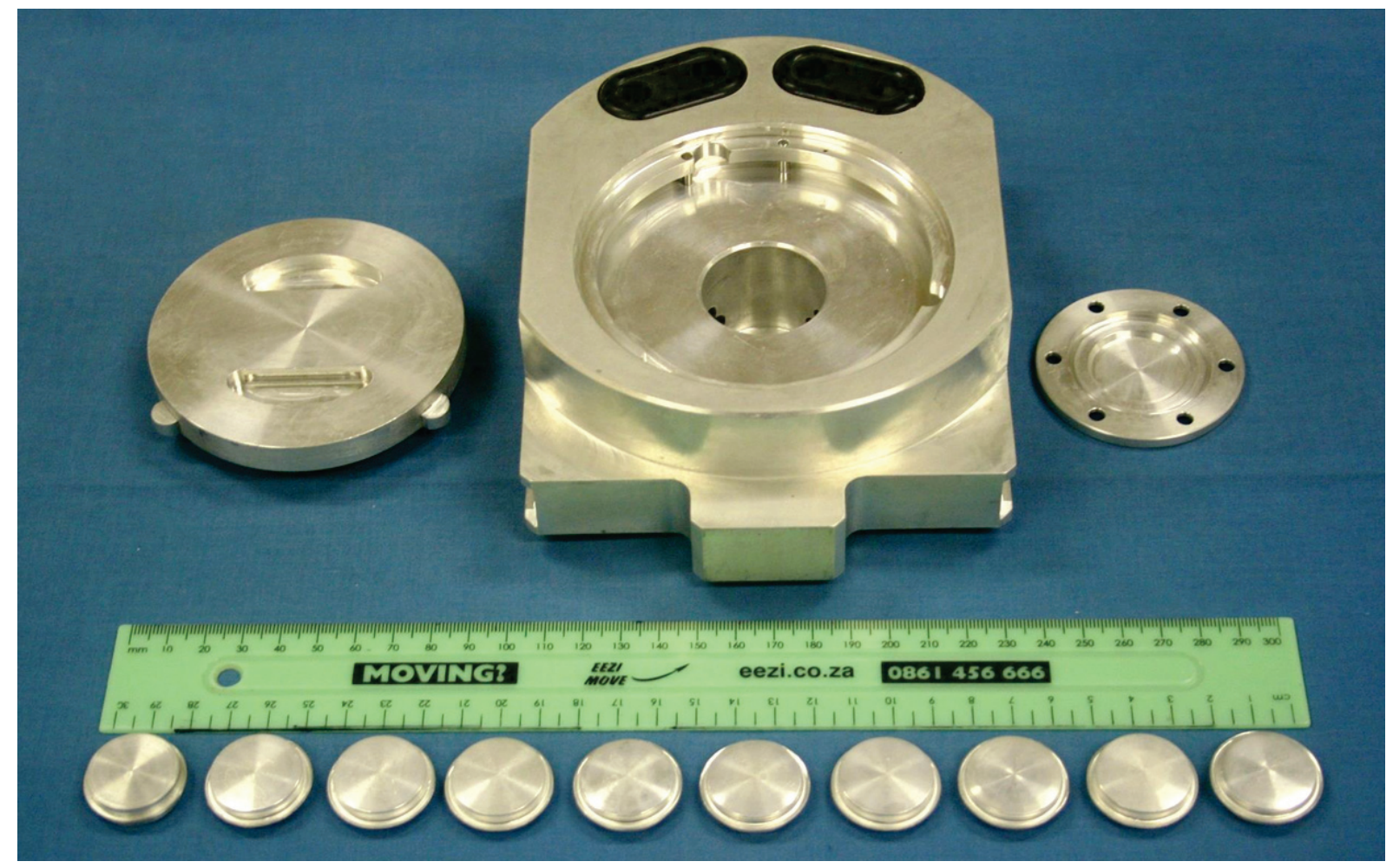

FIGURE 3. Ten Al-encapsulated Th targets and a target holder for high-intensity bombardments. 


\section{ENCAPSULATION USING ELECTRON BEAM WELDING}

Encapsulation by means of cold indentation welding was the sole technique used by iThemba LABS for more than a decade, for the preparation of solid targets for its horizontal-beam target station (Elephant). The decision to develop the vertical-beam target station (VBTS), designed to handle 3 times higher beam currents than the Elephant, placed increasing demands on the targetry. The VBTS is a dedicated target station for the production of longer-lived, high value export radionuclides, such as ${ }^{22} \mathrm{Na},{ }^{68} \mathrm{Ge}$ and ${ }^{82} \mathrm{Sr}$. VBTS targets are bombarded at relatively high beam intensities (nominally $250 \mu \mathrm{A}$ but even up to $300 \mu \mathrm{A}$ ) for extended periods of time. Consequently, the decision was made to use other methods of welding for VBTS target capsules as a measure to ensure a higher target integrity. Also, niobium and stainless steel capsules have to be used for the VBTS targets. These materials are considerably more difficult to cold weld. The VBTS targets are larger than the Elephant targets (outside capsule diameters of $42 \mathrm{~mm}$ and $24 \mathrm{~mm}$, respectively). The larger surface area to remove heat from allows VBTS targets to receive higher beam intensities. At first, we tried to perform in-house tungsten inert gas (TIG) welding but this was not successful as the heat-affected zone (HAZ) proved too large for such small objects. It was subsequently decided to outsource the capsule welding to a commercial company specializing in electron beam (EB) welding. Currently, Nb-encapsulated ${ }^{\text {nat }} \mathrm{Mg}$ targets (for ${ }^{22} \mathrm{Na}$ production) and ${ }^{n a t} \mathrm{Ga}$ targets (for ${ }^{68} \mathrm{Ge}$ production) are prepared in this way.

Figure 4 shows an example of a $\mathrm{Nb}$ capsule for a $\mathrm{Mg}$ target disc. Targetry components such as these are prepared in-house at iThemba LABS, vacuum packed in plastic shrink film, and sent out as complete "kits" for welding. The manufacture of ${ }^{\text {nat }} \mathrm{Ga}$ targets is somewhat more complex. Gallium has a low melting point of only $29.7^{\circ} \mathrm{C}$, i.e. only marginally above typical room temperature. In this case, the "cup" part of the capsule is filled with molten Ga until there is a convex meniscus protruding about $1 \mathrm{~mm}$ above the capsule flange. The filled capsule is afterwards placed in a freezer to solidify the $\mathrm{Ga}$, cooling it down to about $-10^{\circ} \mathrm{C}$. A small milling machine is then used to remove the excess $\mathrm{Ga}$, by machining it down until the Ga surface is level with the $\mathrm{Nb}$ flange surface. The $\mathrm{Nb}$ lid is then placed into position, the whole assembly vacuum packed in shrink film, before returning it back to the freezer. Filled capsules are transported to the premises of the EB welding company in a cooler box, where on arrival they are again stored overnight in a freezer. Prior to the welding, a filled target capsule and its lid is mounted in a CNC collet chuck inside the vacuum chamber of the apparatus and pumped down to a vacuum of less than 0.1 mbar. The electron gun is stationary during welding and the cold capsule is rotated so that the welding speed is approximately $25 \mathrm{~mm} / \mathrm{s}$. The welding is performed at a voltage of $100 \mathrm{kV}$ and a beam current of about $3 \mathrm{~mA}$. The actual welding is quick, only lasting between 5 and 6 seconds. While there is significant local heating on the flanges, the heat transfer is not fast enough to affect the phase of the $\mathrm{Ga}$, which remains frozen during the welding operation. On arrival back at iThemba LABS, each target is visually inspected and individually labelled.

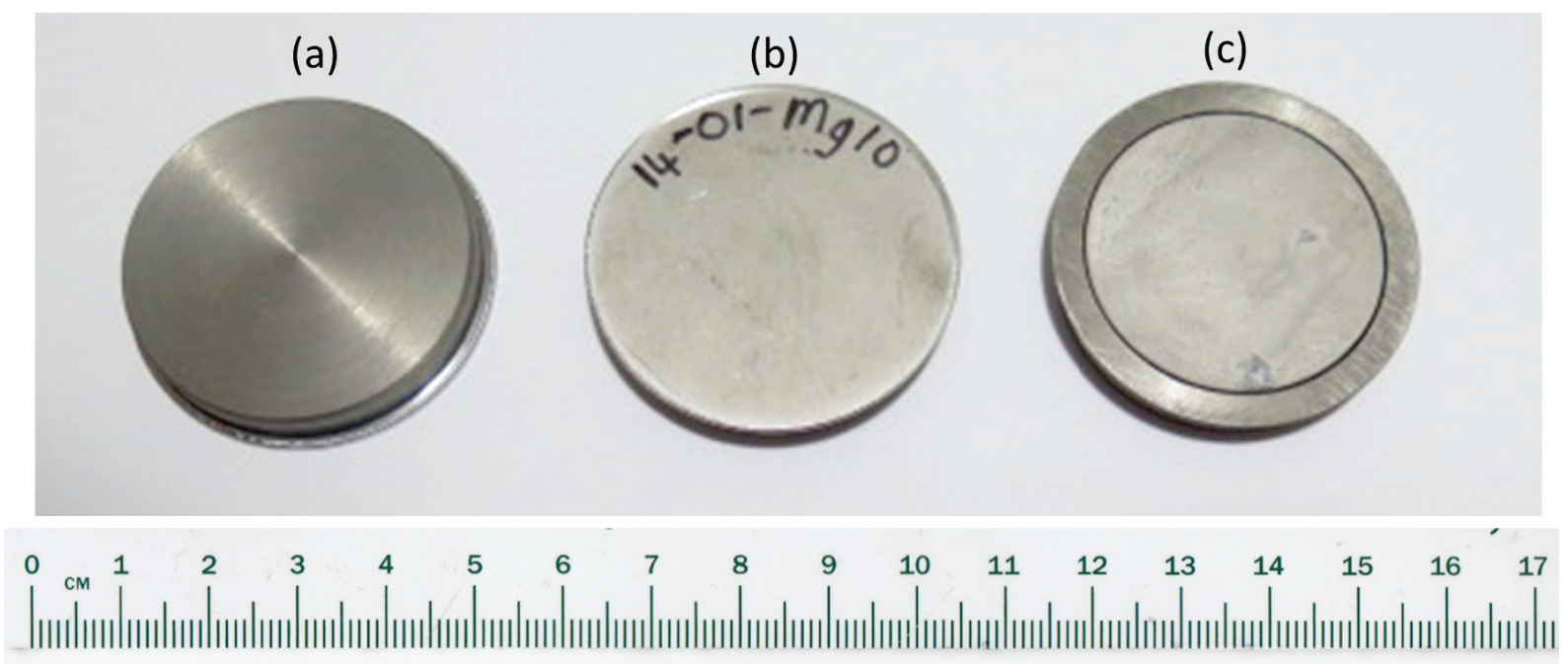

FIGURE 4. Electron-beam-welded niobium capsule for a Mg target: (a) Back view. (b) Front view. (c) The window removed to show the Mg target disc inside. 


\section{ENCAPSULATION USING LASER WELDING}

Capsules for ${ }^{n a t} \mathrm{Rb}$ targets (for ${ }^{82} \mathrm{Sr}$ production) introduced a whole new set of conditions. The capsule material has been specified by one of iThemba LABS's main clients to be type 316 stainless steel, a condition we have to adhere to. The capsule requires a radially mounted 1/8" Swagelok fitting for filling with molten Rb metal (see Fig. 5). Each capsule consists of four components: two identical die-pressed windows made of $0.5 \mathrm{~mm}$ thick stainless steel plate, a ring-shaped body, and the Swagelok fitting. In this case, there is no specific need for the welding to be performed under vacuum. Test batches of capsule components were sent to different specialist companies for welding. As expected, EB welding produced good quality targets. The laser-welded units, however, really stood out as superior. Consequently, it was decided to adopt laser welding for the Rb target-capsule manufacturing - the same service provider has been welding these capsules for iThemba LABS for the past decade. A state-of-the-art Nd-YAG laser welding apparatus equipped with an advanced robotic positioning system is used for this purpose [15].

Empty target capsules are shipped in batches from iThemba LABS to the premises of the client. There they are filled with $\mathrm{Rb}$, sealed and individually labelled, before being sent back to iThemba LABS for bombardment. Once filled, these targets become the property of the client. Rubidium metal has a low melting point of only $39.48{ }^{\circ} \mathrm{C}$ but unlike non-toxic $\mathrm{Ga}$, it has to be handled with much more care. Rubidium is extremely reactive and oxidizes rapidly in air. The filling of these capsules has to be performed in an inert gas atmosphere. The same applies to the decapsulation process after bombardment.
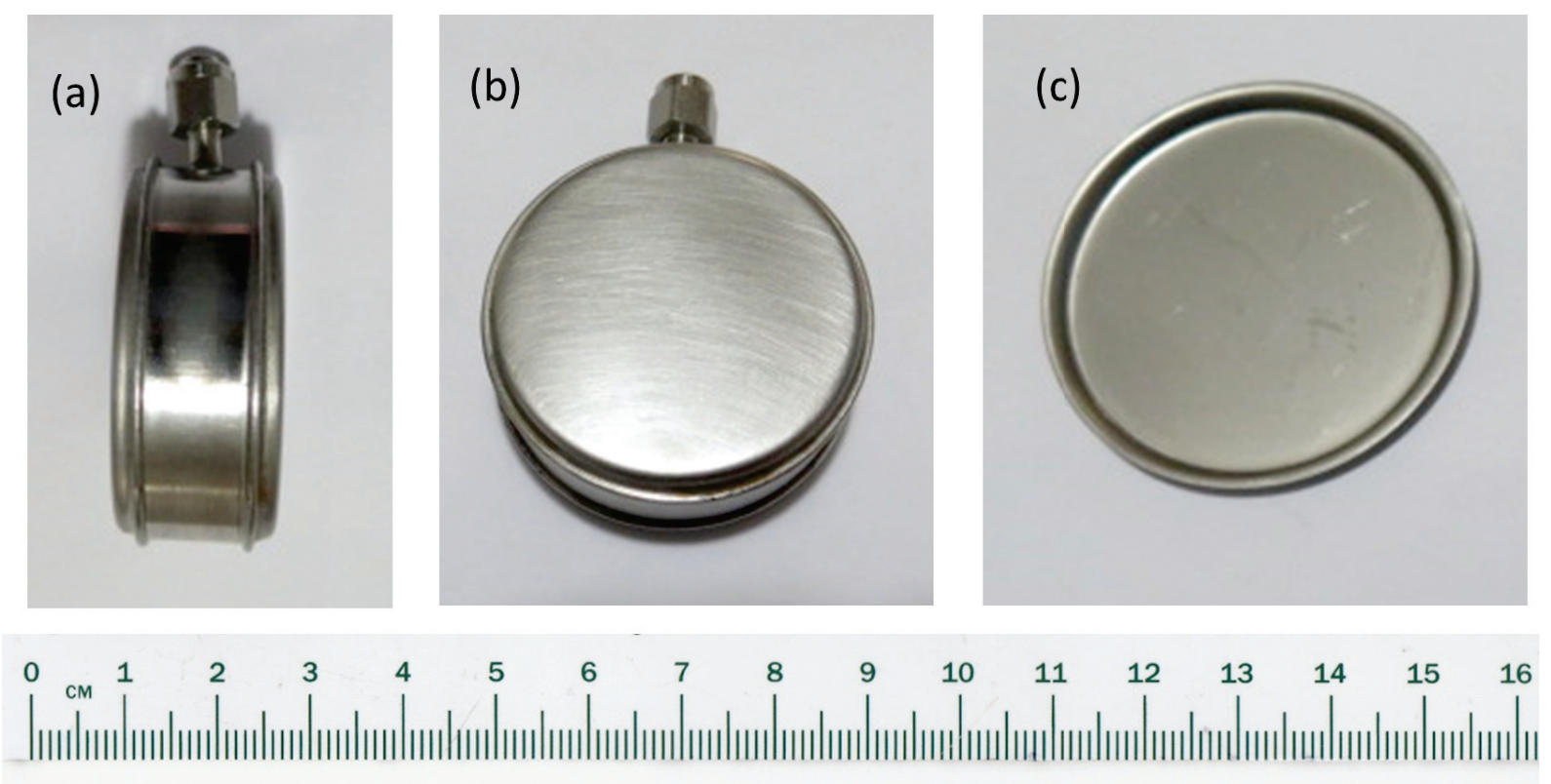

FIGURE 5. Laser-welded stainless steel capsule for a Rb target: (a) Side view. (b) Front view. (c) A single capsule window.

\section{TANDEM TARGETS}

Tandem target configurations are extensively utilized at iThemba LABS. Figure 6 shows a typical VBTS target holder as well as tandem $\mathrm{Rb} / \mathrm{Ga}$ targets for simultaneous ${ }^{82} \mathrm{Rb} /{ }^{68} \mathrm{Ge}$ production, on the Elephant and VBTS, respectively. As already mentioned before, the target diameters for the two stations differ but their thicknesses are similar. Multiple ports for cooling water are provided on the target holders. Each inlet port provides about $30 \mathrm{~L} / \mathrm{min}$ to the target holder at a pressure of nominally 10 bar. The linear flow velocity across a heated target surface is about $16 \mathrm{~m} / \mathrm{s}$. The high flow rate is sufficient to suppress surface boiling. 


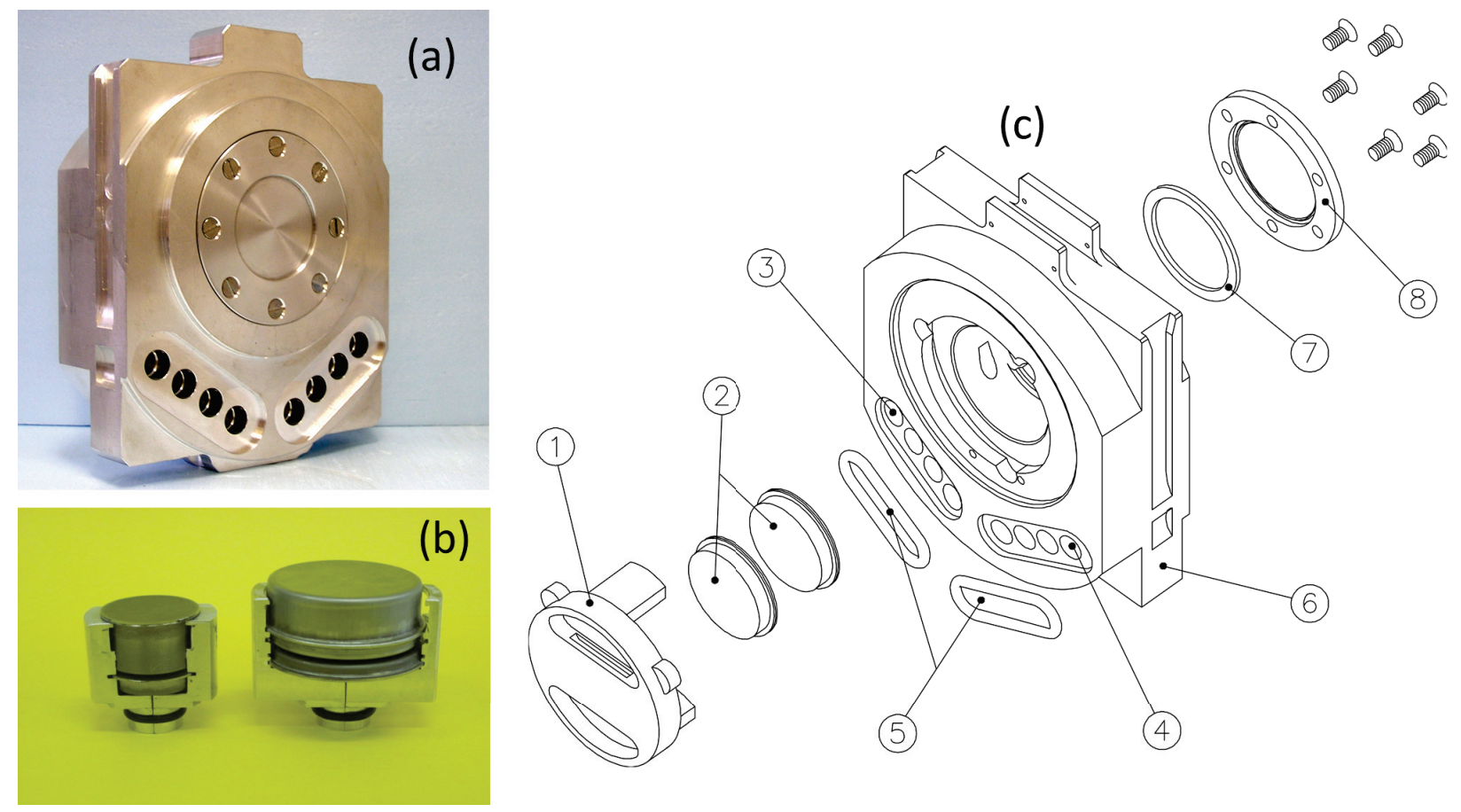

FIGURE 6. (a) A typical VBTS target holder for radionuclide production with a $66 \mathrm{MeV}$ proton beam. (b) Tandem targets for the horizontal-beam target station (LEFT) and vertical-beam target station (RIGHT). (c) Exploded view of a target holder, showing (1) Al bayonet plug that also serves as a beamstop, (2) tandem targets, (3) inlet ports for cooling water, (4) outlet ports for cooling water, (5) molded isobutyl rubber seals, (6) Al target body, (7) metal seal, (8) Al beam entrance window.

\section{CONCLUSION}

Some aspects of the targetry and the methods used at iThemba LABS for target encapsulation have been described. These methods have been in use for many years and proved to be reliable and well suited to the requirements of the radionuclide production programme. Future plans for upgrading the facilities at iThemba LABS include a proposal for a dedicated $70 \mathrm{MeV}$ cyclotron for radionuclide production. Should this become a reality, new target stations will be built, largely following the current philosophy, which we think is a good one. Only horizontal beams will be used, however. Vertical beams have some advantages but also some distinct disadvantages, especially maintenance tasks and repairs on hard-to-reach beamline components. From a targetry perspective, one may expect upward pressure on the beam intensity - some commercial $70 \mathrm{MeV}$ cyclotrons can already deliver dual proton beams of $350 \mu \mathrm{A}$ each. It is no simple task to utilize beams of that intensity, both from a radiation and a target heating perspective. These issues can be handled but require very well-engineered solutions, such as integrated shielding with complementary shielding materials, good electronic interlocks and improved target cooling. The main challenge will be to provide sufficient cooling. Interesting options exist but need further investigation, such as cooling schemes based on submerged multiple jets. We are confident, however, that current encapsulation methods will suffice for future, upgraded targetry.

\section{ACKNOWLEDGMENTS}

This work was financially supported by the National Research Foundation (NRF Grant no. 85507).

\section{REFERENCES}

[1] A. Arzumanov, N. Berdinova, A. Borissenko, G. Chumikov, N. Gorodisskaya, A. Knyazev, V. Koptev, Yu. Popov, G. Sychikov and D. Zheltov, "Status of cyclotron based radioisotopes production in Kazakhstan: 
Report from the laboratory.” In: S.-J. Heselius, J.-O. Lill, J. Rajander (Eds.), Proc. $9^{\text {th }}$ Int. Workshop on Targetry and Target Chemistry, Turku, Finland, 2002. Report ÅA Acc-1, Turku, ISBN 952-12-1383-3; 2004, pp. 92-97. Available from <http://wttc.triumf.ca>.

[2] W. Gelbart and R.R. Johnson, “5th Generation high current solid target irradiation system.” In: J. Steinbach, O. Lebeda, M. Walther (Eds.), Proc. 15 th Int. Workshop on Targetry and Target Chemistry, Prague, Czech Republic, 2014. Published by Helmholtz-Zentrum Dresden-Rossendorf, ISBN 978-3-941405-18-9; 2015, pp. 70-72. Available from <http://wttc.triumf.ca $>$.

[3] F.M. Nortier, J.W. Lenz and P.A. Smith, "Large-scale isotope production with an intense $100 \mathrm{MeV}$ proton beam: Recent target performance experience.” In: D. Rifuggiato, L.A.C. Piazza (Eds.), Proc. 18 ${ }^{\text {th }}$ Int. Conference on Cyclotrons and their Applications, Giardini-Naxos, Sicily, Italy, 2007. Presso la C.D.B. di Ragusa, 2008; pp. 257-259. Available from <http://www.jacow.org >.

[4] H.T. Bach, J.F. Hunter, D.A. Summa, C.J. Stull, E.R. Olivas, M.A. Connors, D.A. Reass, C. Moddrell, F.M. Nortier and K.D. John, "Non-destructive evaluation of $\mathrm{RbCl}$ and $\mathrm{Rb}$ targets in $\mathrm{Sr}-82$ production." In: J. Steinbach, O. Lebeda, M. Walther (Eds.), Proc. $15^{\text {th }}$ Int. Workshop on Targetry and Target Chemistry, Prague, Czech Republic, 2014. Published by Helmholtz-Zentrum Dresden-Rossendorf, ISBN 978-3-941405-18-9; 2015, pp. 63-66. Available from <http://wttc.triumf.ca $>$.

[5] G.F. Steyn, C. Vermeulen, A.H. Botha, J.L. Conradie, J.P.A. Crafford, J.L.G. Delsink, J. Dietrich, H. du Plessis, D.T. Fourie, Z. Kormány, P.F. Rohwer, N.P. Stodart and J.G. de Villiers, "A vertical-beam target station and high-power targetry for the cyclotron production of radionuclides with medium energy protons." Nucl. Instr. and Meth. A 727, 131-144 (2013).

[6] A.B. Sowter, "Cold pressure welding." J. Brit. Inst. Radio Eng. 10, 404-406 (1950).

[7] N. Bay, "Cold pressure welding - The mechanisms governing bonding." ASME J. Eng. Ind. 101, 121-127 (1979).

[8] T. Tabata, S. Masaki and K. Azekura, "Bond criterion in cold pressure welding of aluminium.” J. Mater. Sci. and Technol. 5, 377-381 (1989).

[9] F.M. Nortier, F.J. Haasbroek, S.J. Mills, H.A. Smit, G.F. Steyn, C.J. Stevens, T.F.H.F. van Elst and E. Vorster, "Targetry for the routine bombardment of solid targets at the National Accelerator Centre." In: R. Weinreich (Ed.), Proc. $4^{\text {th }}$ Int. Workshop on Targetry and Target Chemistry, Villigen, Switzerland, 1991. PSIProceedings 92-1; 1992, pp. 60-63. Available from <http://wttc.triumf.ca $>$.

[10] M. Sahin, "Effect of surface roughness on weldability in aluminium sheets joined by cold pressure welding." Ind. Lubr. Tribol. 60, 249-254 (2008).

[11] W. Zhang and N. Bay, "A numerical model for cold welding of metals." CIRP Annals 45, $215-220$ (1996).

[12] W. Zhang and N. Bay, "Cold welding - theoretical modeling of the weld formation." Weld. J. Res. Suppl. October, 417s-420s (1997).

[13] A. Guglielmetti, D. Faccio, R. Bonetti, S.P. Tretyakova, S.V. Dmitriev, A.A. Ogloblin, G.A. Pik-Pichak, N.P. van der Meulen, G.F. Steyn, T.N. van der Walt, C. Vermeulen and D. McGee, "Carbon radioactivity of ${ }^{223} \mathrm{Ac}$ and a search for nitrogen emission.” J. Phys. Conf. Ser. 111, 012050 (2008).

[14] A. Guglielmetti, D. Faccio, G.F. Steyn, R. Bonetti, S.P. Tretyakova, S.V. Shishkin, A.A. Ogloblin, G.A. PikPichak, C. Vermeulen, N.P. van der Meulen, T.N. van der Walt and D. McGee, "Cluster emission in the radioactive decay of ${ }^{223}$ Ac." In: F. Cerutti, A. Ferrari (Eds.), Proc. $12^{\text {th }}$ Int. Conference on Nuclear Reaction Mechanisms, Varenna, Italy, 2009. CERN-Proceedings-2010-001, 2010, pp. 307-315.

[15] G.F. Steyn, C. Vermeulen, E. Isaacs, S. DeWindt, D. Saal, H.P. Burger, C. van Rooyen, F.C. de Beer, H. Knox and J. Isobe, "Development of tandem targets for a vertical beam target station." In: J.M. Link (Ed.), Proc. $12^{\text {th }}$ Int. Workshop on Targetry and Target Chemistry, Seattle, Washington, USA, 2008. University of Washington; 2015, pp. 51-53. Available from <http://wttc.triumf.ca>. 